\title{
A Comparison of Depth Functions in Maximal Depth Classification Rules
}

Olusola Samuel Makinde

Federal University of Technology, Akure, Nigeria, osmakinde@futa.edu.ng

Adeyinka Damilare Adewumi

Federal University of Technology Akure, Nigeria, adewumiadeyinkad@yahoo.com

Follow this and additional works at: http://digitalcommons.wayne.edu/jmasm

Part of the Applied Statistics Commons, Social and Behavioral Sciences Commons, and the Statistical Theory Commons

\section{Recommended Citation}

Makinde, O. S. \& Adewumi, A. D. (2017). A comparison of depth functions in maximal depth classification rules. Journal of Modern Applied Statistical Methods, 16(1), 388-405. doi: 10.22237/jmasm/1493598120

This Regular Article is brought to you for free and open access by the Open Access Journals at DigitalCommons@WayneState. It has been accepted for inclusion in Journal of Modern Applied Statistical Methods by an authorized editor of DigitalCommons@WayneState. 


\section{A Comparison of Depth Functions in Maximal Depth Classification Rules}

\author{
Olusola Samuel Makinde \\ Federal University of Technology \\ Akure, Nigeria
}

\author{
Adeyinka Damilare Adewumi \\ Federal University of Technology \\ Akure, Nigeria
}

Data depth has been described as alternative to some parametric approaches in analyzing many multivariate data. Many depth functions have emerged over two decades and studied in literature. In this study, a nonparametric approach to classification based on notions of different data depth functions is considered and some properties of these methods are studied. The performance of different depth functions in maximal depth classifiers is investigated using simulation and real data with application to agricultural industry.

Keywords: classification rules, data depth, error rates, non-parametric approach, symmetry

\section{Introduction}

Classification is a practical subject in statistics. It aims at assigning an unclassified observation to one of several groups or populations on the basis of some measurement. Anderson (1984) described classification problem as a problem of statistical decision-making. However, classical multivariate analysis has relied heavily on the assumption of normality in data presentation and analysis. Among the classification methods that rely heavily on distribution assumption are Bayes rule (Welch, 1938), linear discriminant analysis and quadratic linear discriminant analysis (Anderson, 1984), and independence rule (Dudoit, Fridlyand \& Speed, 2002). Research has shown that most of the data acquired nowadays do not satisfy normality assumption. Similarly, some parametric approaches are prone to the effect of outlying observations. This gives nonparametric approach to classification an edge over parametric methods.

Olusola Samuel Makinde is a Lecturer in the Department of Statistics. Email at osmakinde@futa.edu.ng. Adeyinka Damilare Adewumi is a graduate of the Department of Statistics. Email at adewumiadeyinkad@yahoo.com. 
Other methods in literature include support vector machine (Vapnik, 1998; Cortes \& Vapnik, 1995), nearest neighbour rule (Cover \& Hart, 1967), classification rules based on distance functions (Chan \& Hall, 2009; Hall, Titterington \& Xue, 2009), classifiers based on distribution functions of rank outlyingness (Makinde \& Chakraborty, 2015).

Data depth is a way to measure the depth or outlyingness of a given point with respect to a multivariate data cloud or its underlying distribution (Liu, Singh \& Parelius, 1999). It gives rise to a natural centr-outward ordering of the sample points in $\mathbb{R}^{d}$. This ordering gives rise to new and easy ways to quantify many complex multivariate features of the underlying distribution, including location, quantiles, scale, skewness and kurtosis. Liu (1990) introduced a notion of simplicial depth and corresponding estimators of location, and formulated a quality index with simplicial depth, Mahalanobis depth and majority depth. Koshevoy \& Mosler (1997) introduced a notion of zonoid depth while Fraiman, Meloche \& García-Escudero (1999) introduced a likelihood type depth function. Rousseeuw \& Hubert (1999) introduced a notion of regression depth. Liu, Singh $\&$ Parelius (1999) considered some examples of depth functions and developed methodology for their practical applications.

Classification rule based on data depth is considered in the current study. Data depth is formally defined based on Zuo \& Serfling (2000a) and examples of depth functions are presented. In reality, an important question that arises in almost all fields where supervised learning is employed is that which of the depth functions should be employed. Classification rules based on the depth functions are defined and properties of the classification rules are presented. Evaluation of the classification rule, accounting for performance of various depth functions are presented based on numerical examples.

\section{Notions of Statistical Depth Functions}

Definition 1 (Zuo \& Serfling, 2000a). Let the mapping $D(. ;):. \mathbb{R}^{\mathrm{d}} \times \mathcal{F} \rightarrow \mathbb{R}$ be bounded and non-negative, and satisfy:

i. $\quad D\left(\mathbf{A x}+\mathbf{b}, F_{\mathbf{A X}+\mathbf{b}}\right)=D\left(\mathbf{x}, F_{\mathbf{X}}\right)$ holds for any random vector $\mathbf{X} \in \mathbb{R}^{d}$ and any $d \times d$ nonsingular matrix $\mathbf{A}$, and any $d$ dimensional vector $\mathbf{b}$.

ii. $\quad D(\boldsymbol{\theta}, F)=\sup _{\mathbf{x} \in \mathbb{R}^{d}} D(\mathbf{x}, F)$ holds for any $F \in \mathcal{F}$ having centre $\boldsymbol{\theta}$.

iii. For any $F \in \mathcal{F}$ having deepest point $\boldsymbol{\theta}, D(\mathbf{x}, F) \leq D(\boldsymbol{\theta}+\alpha(\mathbf{x}-\boldsymbol{\theta}), F)$ holds for $\alpha \in[0,1]$; and 


\section{DEPTH FUNCTIONS IN MAXIMAL DEPTH CLASSIFICATION RULES}

iv. $\quad D(\mathbf{x}, F) \rightarrow 0$ as $\|\mathbf{x}\| \rightarrow \infty$, for each $F \in \mathcal{F}$.

Then $D(., F)$ is called a statistical depth function.

From Definition 1, the first property describes invariance of depth function under general affine transformation of the data. That is, the depth of any observation in $\mathbb{R}^{d}$ should not depend on the scale of the underlying measurement or underlying coordinate system. The second property implies that depth value attains its maximum value at the point of symmetry for symmetric distributions. The third property implies that the depth value decreases monotonically as vector $\mathbf{x}$ moves away from its most central point while the fourth property implies that the depth value of $\mathbf{x}$ vanishes (tend to zero) as Euclidean norm of $\mathbf{x}$ approaches infinity.

The depth functions in literature include

1. Mahalanobis Depth (MhD). Mahalanobis (1936); Liu \& Singh (1993) defined the depth of an observation $\mathbf{x}$ with respect to the distribution $\mathrm{F}$ as

$$
\operatorname{MhD}(\mathbf{x}, F)=\left[1+O\left(\mathbf{x}, \boldsymbol{\mu}_{F}, \Sigma_{F}\right)\right]^{-1}
$$

where $O\left(\mathbf{x}, \boldsymbol{\mu}_{F}, \Sigma_{F}\right)=\left(\mathbf{x}-\boldsymbol{\mu}_{F}\right)^{\prime} \Sigma_{F}^{-1}\left(\mathbf{x}-\boldsymbol{\mu}_{F}\right), \boldsymbol{\mu}_{F}$ and $\boldsymbol{\Sigma}_{F}$ are the mean vector and dispersion matrix of $F$ respectively. The sample version of $M h D$ is obtained by replacing $\boldsymbol{\mu}_{F}$ and $\Sigma_{F}$ with their estimates.

2. Zonoid Depth (ZD). Dyckerhoff et al. (1996) defined a zonoid depth as

$$
Z D(\mathbf{x}, F)=\sup \left\{\alpha: \mathbf{x} \in D_{\alpha}\left(\mathbf{X}_{1}, \ldots, \mathbf{X}_{n}\right)\right\}
$$

where $D_{\alpha}\left(\mathbf{X}_{1}, \ldots, \mathbf{X}_{n}\right)=\sum_{i=1}^{n} \lambda_{i} \mathbf{X}_{i}, \sum_{i=1}^{n} \lambda_{i}=1, \lambda_{i} \geq 0$, and $\alpha \lambda_{i} \leq \frac{1}{n}$ for all $i$.

3. Half-Space Depth (HD). Tukey (1975) defined half-space depth of a point $\mathbf{x} \in \mathbb{R}^{d}$ with respect to $F$ as the minimum probability mass carried by any closed half-space containing $\mathbf{x}$, Mathematically,

$$
H D(\mathbf{x}, P)=\inf _{H}[P(H)]
$$


where $H$ is a closed halfspace in $\mathrm{R}^{d}$ and $\mathbf{x} \sim H$.

4. Oja Depth (OD). Oja (1983) defined the depth of $\mathbf{x} \in \mathbb{R}^{d}$ with respect to $F$ as

$$
O D(F ; \mathbf{x})=[1+O(\mathbf{x}, F)]^{-1}
$$

where $O(\mathbf{x}, F)=E_{F}\left(\operatorname{Volume}\left(S\left[\mathbf{x}, \mathbf{X}_{1}, \ldots, \mathbf{X}_{d}\right]\right)\right), \quad S\left[\mathbf{x}, \mathbf{X}_{1}, \ldots, \mathbf{X}_{d}\right]$ is a closed simplex with vertices $\mathbf{x}$ and $d$ random observations $\mathbf{X}_{1}, \ldots, \mathbf{X}_{d}$ from $F$.

5. Simplicial Depth (SD). Liu (1990) defined simplicial depth of $\mathbf{x} \in \mathbb{R}^{d}$ with respect to $F$ as

$$
S D(F ; \mathbf{x})=\mathrm{P}\left(\mathbf{x} \in S\left[\mathbf{x}, \mathbf{X}_{1}, \ldots, \mathbf{X}_{d+1}\right]\right)
$$

where $S\left[\mathbf{x}, \mathbf{X}_{1}, \ldots, \mathbf{X}_{d+1}\right]$ is a closed simplex formed by $(d+1)$ random observation from $F$. The sample version of $S D(F ; \mathbf{x})$ is obtained by replacing $F$ in $S D(F ; \mathbf{x})$ by $F_{n}$.

6. Projection Depth (PD). Donoho \& Gasko (1982) defined the depth of $\mathbf{x}$ with respect to $F$ as the worst case outlyingness of $\mathbf{x}$ with respect to one dimensional median in any one-dimensional projection.

$$
P D(F ; \mathbf{x})=(1+O(\mathbf{x}, F))^{-1}
$$

where $O(\mathbf{x}, F)=\sup _{\|u\|=1} \frac{\mathbf{u}^{\prime} \mathbf{x}-\operatorname{Med}\left(F_{u}\right)}{\operatorname{MAD}\left(F_{u}\right)}, \quad F_{u} \quad$ is the distribution $\mathbf{u}^{\prime} \mathbf{X}$, $\operatorname{Med}\left(F_{u}\right)$ is the median of $F_{u}, \operatorname{MAD}\left(F_{u}\right)$ is the median absolute deviation of $F_{u}$ and $X \sim F$. The sample version of $P D(F ; \mathbf{x})$ is obtained by replacing the median and $M A D$ with their sample estimates.

7. Likelihood Depth (LD). Fraiman, Meloche \& García-Escudero (1999) defined the depth of $\mathbf{x}$ with respect to $F$ simply as its probability density, that is, $L D(F ; \mathbf{x})=f(\mathbf{x})$, and the empirical version 
can be any consistent density estimate at $\mathbf{x}$, for example, the kernel density estimate.

8. Spatial Depth (SPD). Serfling (2002) defined spatial depth of any observation $\mathbf{x}$ with respect to $F$ as

$$
\operatorname{SBD}(\mathbf{x}, F)=1-\left\|E_{F}\left(\frac{\mathbf{x}-\mathbf{X}}{\|\mathbf{x}-\mathbf{X}\|}\right)\right\|
$$

where $X \sim F$.

9. Simplicial volume depth (SVD). Zuo \& Serfling (2000a, b) expressed SVD of an observation $\mathbf{x}$ with respect to $F$ as

$$
S V D(\mathbf{x}, \delta, F)=\left\{1+E_{F}\left(\frac{\nabla\left(\mathbf{x}, \mathbf{X}_{1}, \ldots, \mathbf{X}_{d}\right)}{\left|\Sigma_{F}\right|^{\frac{1}{2}}}\right)^{\delta}\right\}^{-1}
$$

where $\mathbf{X}_{1}, \ldots, \mathbf{X}_{d}$ are independent and identically distributed observations from $F, \boldsymbol{\nabla}\left(\mathbf{x}, \mathbf{X}_{1}, \ldots, \mathbf{X}_{d}\right)$ is the volume of the $d$ dimensional simplex formed by $\mathbf{x}$ and $\Sigma_{F}$ is the scatter matrix of the distribution F.

10. Majority Depth (MJD). Liu \& Singh (1993) defined the depth of $\mathbf{x}$ with respect to $F$ as the probability that $\mathbf{x}$ belongs to the major side (i.e. the half-space with the larger probability measure) of a random hyperplane passing through the data points in $\mathbb{R}^{d}$.

Other depth functions include regression depth (Rousseeuw \& Hubert, 1999). Gao (2003) defined another depth function based on square of spatial outlyingness function. Few of these depth functions satisfy all the four properties in Definition 1 while others satisfy some of the properties. See Zuo \& Serfling (2000a; 2000b) for detail. 


\section{Classification Rule}

The goal of any classification rule is to find a rule or tool that enables us to assign an observation $\mathbf{x} \in \mathbb{R}^{d}$ to one of the several competing groups (or classes). One can define a classification rule based on depth functions. It is easy to observe that data depth gives an idea on how outlying an observation $\mathbf{x}$ is with respect to the distribution $F$. If $\mathbf{x}$ is a central observation, its depth value will be large. On the other hand, if $\mathbf{x}$ is an extreme observation, its depth value will be small. Thus a small depth value may suggest a deviation of $\mathbf{x}$ from $F$.

Ghosh \& Chaudhuri (2005) proposed a classification rule based on simple idea of assigning a new observation to any of the $\mathrm{J}$ competing classes, for which it attains maximal depth value. It is expressed as:

$$
D\left(F_{k}, \mathbf{x}\right)=\arg \max _{1 \leq j \leq J} D\left(F_{j}, \mathbf{x}\right)
$$

where $F_{k}$ is the distribution of $k^{\text {th }}$ class and $1 \leq j \leq J$.

Let us consider two classes for simplicity. Suppose $\pi_{j}$ has multivariate distribution with mean vector $\boldsymbol{\mu}_{j}$ and covariance matrix $\boldsymbol{\Sigma}_{j}, j=1,2$. For $\boldsymbol{\Sigma}_{1}=\boldsymbol{\Sigma}_{2}=\boldsymbol{\Sigma}$, the classification rule in (1) can be expressed as

$$
\text { Assign } \mathbf{x} \text { to } F \text { if } D(F, \mathbf{x})>D(G, \mathbf{x}) \text {, and to } G \text { if otherwise. }
$$

It is straightforward to show that a depth function can be expressed in terms of probability density function of the competing distribution. This result is presented by a Lemma below:

Lemma 1. Let $F_{j}$ be spherically symmetric distributions with density functions of the form

$$
f_{j}(\mathbf{x})=\left|\Sigma_{j}\right|^{-\frac{1}{2}} h\left(\mathbf{x}-\boldsymbol{\theta}_{j}\right)^{\prime}\left(\mathbf{x}-\boldsymbol{\theta}_{j}\right)
$$

for some strictly decreasing, continuous, non-negative scalar function $\mathrm{h}$. Then for any of the depth functions OD and SPD,

$$
f_{j}(\mathbf{x})=\omega\left(D\left(F_{j}, \mathbf{x}\right)\right)
$$




\section{DEPTH FUNCTIONS IN MAXIMAL DEPTH CLASSIFICATION RULES}

for some increasing function $\omega$.

Suppose a random vector $\mathbf{X}$ in $\mathbb{R}^{d}$ is elliptically distributed such that its density is of the form $f(\mathbf{x})=|\boldsymbol{\Sigma}|^{-1 / 2} h\left((\mathbf{x}-\boldsymbol{\theta})^{\prime} \boldsymbol{\Sigma}^{-1}(\mathbf{x}-\boldsymbol{\theta})\right)$, then $D(F, \mathbf{x})$ can be expressed as a function of $(\mathbf{x}-\boldsymbol{\theta})^{\prime} \boldsymbol{\Sigma}^{-1}(\mathbf{x}-\boldsymbol{\theta})$. This result is presented formally by a Lemma below:

Lemma 2.

Let $F_{j}$ be elliptically symmetric distributions with density functions of the form

$$
f_{j}(\mathbf{x})=\left|\Sigma_{j}\right|^{-\frac{1}{2}} h\left(\left(\mathbf{x}-\boldsymbol{\theta}_{j}\right)^{\prime} \boldsymbol{\Sigma}_{j}^{-1}\left(\mathbf{x}-\boldsymbol{\theta}_{j}\right)\right)
$$

for some strictly decreasing, continuous, non-negative scalar function $h$. Then for any of the depth functions detailed earlier, except OD and SPD,

$$
f_{j}(\mathbf{x})=\omega\left(D\left(F_{j}, \mathbf{x}\right)\right)
$$

where $\Sigma_{j}$ is not a constant multiple of identity matrix for some increasing function $\omega$.

The optimal rule, Bayes rule, assigns an observation to the class or distribution with highest posterior probability. That is, assign $\mathbf{x}$ to $j^{\text {th }}$ class class if $p_{j} f_{j}(\mathbf{x})$ is the highest, where $p_{j}$ is the prior probability of the $j^{\text {th }}$ class. Based on the results of Lemmas 1 and 2, it is straightforward to show that maximum depth classifiers are Bayes rules under necessary conditions.

Theorem 1. Suppose the conditions of Lemmas 1 and 2 hold on all the depth functions defined earlier. Then the classifier defined in (1) is Bayes rule if competing distributions have equal covariance matrices and prior probabilities.

In practice, a depth function may not be completely known and so need to be estimated based on sample and then define the empirical version of the classification rule based on the empirical depth function. The empirical depth function based on sample is denoted by $D\left(F_{n}, \mathbf{x}\right)$. To show the consistency of empirical depth functions, it is desirable to establish the almost sure convergence of empirical depth functions to its population counterpart. 
Theorem 2. Suppose $D\left(F_{n}, \mathbf{x}\right)$ is an empirical depth function based on $\mathbf{X}_{1}, \mathbf{X}_{2}, \ldots, \mathbf{X}_{n}$. Let $D(F, \mathbf{x})$ be a population depth function of any random vector $\mathbf{x}$. Then for any $\mathbf{x}$ in the support of $F$,

$$
\sup _{\mathbf{x}}\left|D\left(F_{n}, \mathbf{x}\right)-D(F, \mathbf{x})\right| \rightarrow 0, n \rightarrow \infty
$$

The almost sure convergence of half-space depth has been established in Donoho \& Gasko (1992), simplicial depth in Liu (1990). Liu \& Singh (1993) has shown almost sure convergence of Mahalanobis depth and majority depth while Zuo \& Serfling (2000b) proved convergence of projection depth. The almost sure convergence of spatial depth follows from Koltchinskii's (1997) work on the convergence of the empirical spatial rank function to its population version. Convergence of the empirical classification rule to population version follows from Theorem 2 .

\section{Evaluation of Classification methods}

One way of evaluating the performance of a classifier is to compute its associated misclassification probability. In a two class classification problem, one can define a misclassification probability as

$$
\Delta=p_{1} P(D(F, \mathbf{x})<D(G, \mathbf{x}) \mid \mathbf{x} \in F)+p_{2} P(D(F, \mathbf{x})>D(G, \mathbf{x}) \mid \mathbf{x} \in G)
$$

The empirical version of the probability of misclassification or error rate, denoted by $\hat{\Delta}$, can be defined as

$$
\begin{aligned}
\hat{\Delta} & =\frac{p_{1}}{n} \sum_{i=1}^{n} I\left\{D\left(\hat{F}, \mathbf{x}_{i}\right)<D\left(\hat{G}, \mathbf{x}_{i}\right) \mid \mathbf{x} \in F\right\} \\
& +\frac{p_{2}}{m} \sum_{i=1}^{m} I\left\{D\left(\hat{F}, \mathbf{x}_{i}\right)>D\left(\hat{G}, \mathbf{x}_{i}\right) \mid \mathbf{x} \in F\right\}
\end{aligned}
$$

Under the conditions of Theorems 1 and 2, it is straightforward to show that $\hat{\Delta}$ is a Bayes risk. 


\section{DEPTH FUNCTIONS IN MAXIMAL DEPTH CLASSIFICATION RULES}

\section{Simulation Study}

As illustration of the performance of maximum depth classification methods, consider the following example. Let populations $\pi_{1}$ and $\pi_{2}$ be bivariate spherically symmetric with centres of symmetry $\boldsymbol{\mu}_{1}$ and $\boldsymbol{\mu}_{2}$, and covariance matrices, $\boldsymbol{\Sigma}_{1}$ and $\Sigma_{2}$, respectively. Assume that the prior probabilities of $\pi_{1}$ and $\pi_{2}$ are equal. Suppose $\mathbf{X}_{1}, \mathbf{X}_{2}, \ldots, \mathbf{X}_{n}$ is a random sample from $\pi_{1}$ and $\mathbf{Y}_{1}, \mathbf{Y}_{2}, \ldots, \mathbf{Y}_{m}$, a random sample from $\pi_{2}$. New random vectors $\mathbf{Z}_{1}, \mathbf{Z}_{2}, \ldots, \mathbf{Z}_{m}$ from $\pi_{1}$ and $\mathbf{Z}_{m+1}, \mathbf{Z}_{m+2}, \ldots, \mathbf{Z}_{2 m}$ from $\pi_{2}$ are generated and sample sizes $n$ and $m$ are taken to be 100. $\boldsymbol{\mu}_{1}$ and $\boldsymbol{\mu}_{2}$ are chosen to be $\left(\begin{array}{ll}0 & 0\end{array}\right)^{T}$ and $\left(\begin{array}{ll}\delta & 0\end{array}\right)^{T}$ respectively for $\delta \in\left[\begin{array}{l}-2,2\end{array}\right]$ and $\boldsymbol{\Sigma}_{1}=\boldsymbol{\Sigma}_{2}=\mathbf{I}_{2}$. The simulation size is taken to be 1000. Different depth functions are considered for some competing distributions. The distributions are bivariate normal distributions and bivariate Laplace distributions. For computation of likelihood depth, Gaussian kernel is used with turning parameter $(=0.3)$. $R$ Package fda.usc is used for computing projection and likelihood depth. $R$ Package depth is used for computing Oja depth, simplicial depth and half-space depth while $R$ Package ddalpha is used for computing simplicial volume depth, Mahalanobis depth and Zonoid depth.

Estimates of misclassification probabilities are less in bivariate normally distributed samples than bivariate Laplace samples, as shown in Figure 1. It is observed from Figures 2 and 3 that maximal depth classification rule based on half-space depth outperforms others when the distinction between competing distributions is not wide. That is, when $\boldsymbol{\mu}_{1}-\boldsymbol{\mu}_{2} \rightarrow \mathbf{0}$. The distinction between competing distributions becomes clear as $\boldsymbol{\mu}_{1}-\boldsymbol{\mu}_{2}$ moves away from $\mathbf{0}$ and the performance of various depth functions becomes equivalent. It is noted that exact computation of half-space depth and simplicial depth functions is feasible only in $\mathbb{R}^{3}$ and $\mathbb{R}^{2}$ respectively. Cuesta-Albertos \& Nieto-Reyes (2010) suggested a modified version of half-space depth for functional data, as extension of multivariate set-up. The performance of empirical likelihood depth based on kernel estimator of probability density function depends on the choice of kernel function and turning parameter. It is observed that spatial depth and Oja depth are not invariant under general affine transformation. Makinde (2017) considered various affine invariant versions of spatial rank, a related notion to spatial depth. Robustness of spatial rank (a straightforward extension of spatial depth) against deviation from notion of elliptical symmetry is demonstrated in Makinde and Chakraborty (2015).

Maximum depth classification rule is compared with some classification methods, which include linear discriminant analysis (LDA), $k$-nearest neighbor 


\section{MAKINDE \& ADEWUMI}

rule $(\mathrm{kNN})$ and support vector machine (SVM); using the above setting for $\delta=1,2$. Table 1 below presents performance of classifiers. It is observed from the table that maximum depth classifiers based on half-space depth has the best performance among the depth based procedures, linear discriminant analysis, $k$-nearest neighbor rule and support vector machine. It has the least mean error rates when the competing distributions are normal and Laplace. Next to halfspace depth among the depth functions for maximum depth classification rule is zonoid depth.

However, zonoid depth is not robust against outlying observations in the data cloud. LDA performs well compared with $\mathrm{kNN}$ and SVM. It is noted that linear discriminant analysis is Bayes (optimal) rule when competing distributions are multivariate normal. Hence maximum depth classifiers based on half-space depth is a better alternative to the known parametric classification methods, e.g. LDA.

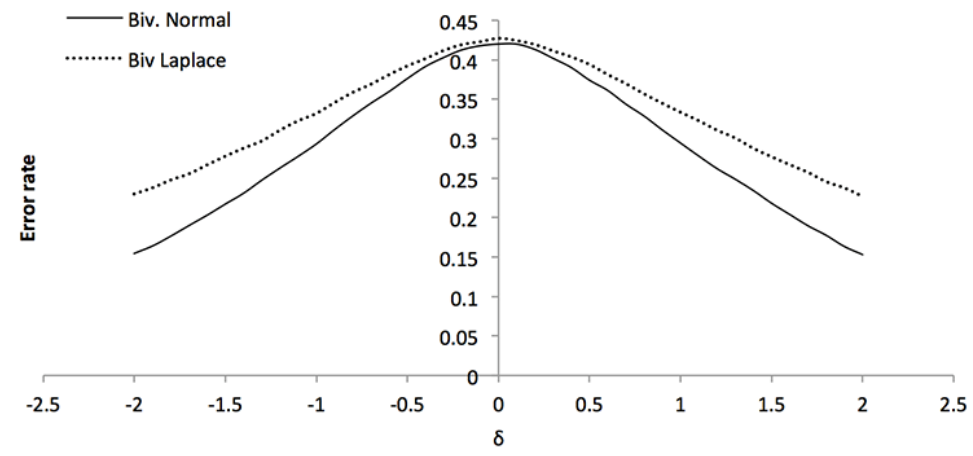

Figure 1. Comparison of error rates associated with half space depth for normally distributed samples and Laplace distributed samples. 


\section{DEPTH FUNCTIONS IN MAXIMAL DEPTH CLASSIFICATION RULES}

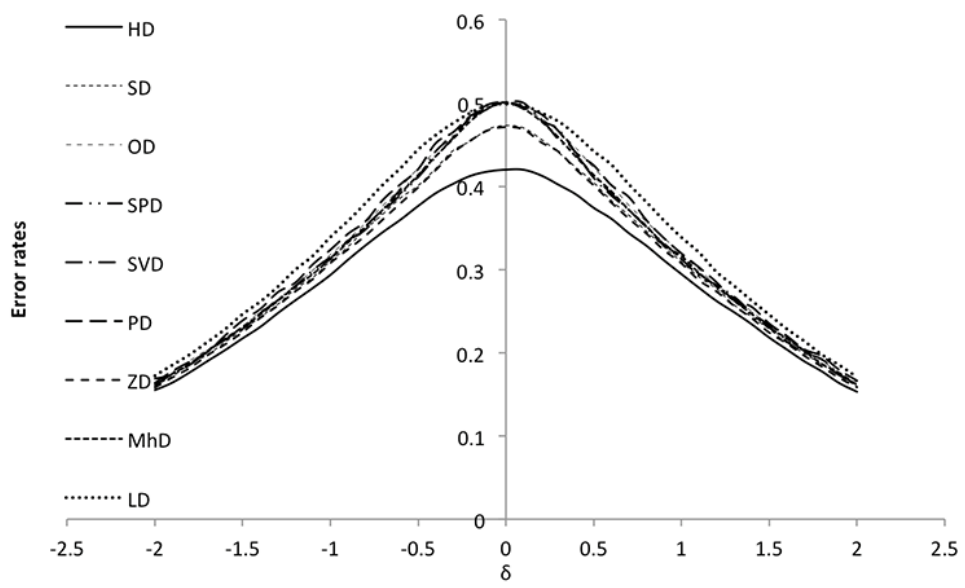

Figure 2. Comparison of depth functions in classification based on error rates for normally distributed samples

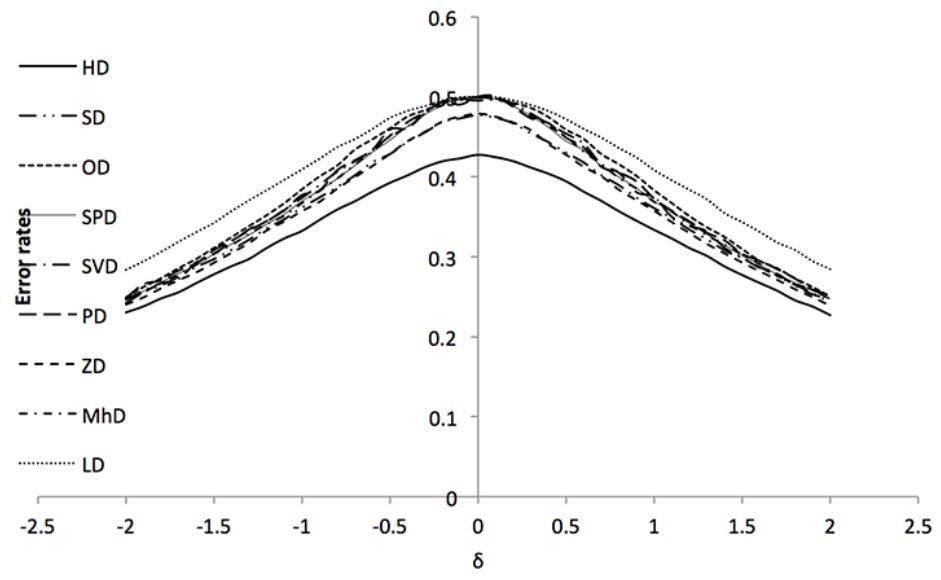

Figure 3. Comparison of depth functions in classification based on error rates Laplace distributed samples. 
Table 1. Comparison of mean error rates of classifiers when competing distributions differ in location.

\begin{tabular}{|c|c|c|c|c|c|c|c|c|c|c|c|c|c|}
\hline \multirow[b]{2}{*}{ Distribution } & \multirow[b]{2}{*}{$\delta$} & \multicolumn{9}{|c|}{ Maximal Depth Classifiers } & \multirow[b]{2}{*}{ LDA } & \multirow[b]{2}{*}{ kNN } & \multirow[b]{2}{*}{ SVM } \\
\hline & & HD & SD & OD & SPD & SVD & PD & ZD & MhD & LD & & & \\
\hline \multirow{2}{*}{ Biv normal } & 1 & 0.295 & 0.309 & 0.318 & 0.313 & 0.313 & 0.319 & 0.307 & 0.316 & 0.334 & 0.315 & 0.356 & 0.316 \\
\hline & 2 & 0.153 & 0.160 & 0.165 & 0.162 & 0.161 & 0.167 & 0.159 & 0.161 & 0.167 & 0.161 & 0.181 & 0.167 \\
\hline \multirow{2}{*}{ Biv Laplace } & 1 & 0.334 & 0.361 & 0.383 & 0.369 & 0.375 & 0.373 & 0.357 & 0.369 & 0.413 & 0.377 & 0.410 & 0.381 \\
\hline & 2 & 0.227 & 0.243 & 0.250 & 0.248 & 0.248 & 0.253 & 0.239 & 0.246 & 0.287 & 0.246 & 0.273 & 0.257 \\
\hline
\end{tabular}

Table 2. Comparison of mean error rates of classifiers when competing distributions differ in location and scale.

\begin{tabular}{|c|c|c|c|c|c|c|c|c|c|c|c|c|}
\hline \multicolumn{13}{|c|}{ Maximal Depth Classifiers } \\
\hline & HD & SD & OD & SPD & SVD & PD & ZD & MhD & LD & QDA & kNN & SVM \\
\hline Biv normal & 0.382 & 0.386 & 0.500 & 0.387 & 0.389 & 0.386 & 0.389 & 0.389 & 0.166 & 0.142 & 0.209 & 0.148 \\
\hline Biv Laplace & 0.410 & 0.417 & 0.500 & 0.418 & 0.421 & 0.419 & 0.421 & 0.418 & 0.255 & 0.214 & 0.282 & 0.214 \\
\hline
\end{tabular}

Table 3. Comparison of computation time of classifiers for bivariate Laplace distributions.

\begin{tabular}{lrrrrrrrrrrrr} 
& \multicolumn{10}{c}{ Maximal Depth Classifiers } & & \\
& HD & SD & OD & SPD & SVD & PD & ZD & MhD & LD & QDA & kNN & SVM \\
\hline Time (seconds) & 0.12 & 0.12 & 0.14 & 0.52 & 15.84 & 5.97 & 0.34 & 0.32 & 1.39 & 0.08 & 0.05 & 0.31 \\
\hline
\end{tabular}

Only populations which are separated by location are considered so far. Table 2 presents a comparison of proportions of misclassification of depth based procedures, quadratic discriminant analysis $(\mathrm{kNN})$ and SVM when competing populations have different location vectors and covariance matrices.

Suppose the mean vectors and covariance matrices of $\pi_{1}$ and $\pi_{2}$ are $\left(\boldsymbol{\mu}_{1}=\left(\begin{array}{ll}0 & 0\end{array}\right)^{T}, \boldsymbol{\Sigma}_{1}=\mathbf{I}_{2}\right)$ and $\left(\boldsymbol{\mu}_{2}=\left(\begin{array}{ll}2 & 0\end{array}\right)^{T}, \boldsymbol{\Sigma}_{2}=9 \mathbf{I}_{2}\right)$, respectively. It is well known that QDA is an optimal rule when competing populations are normally distributed and differ in location and scale. Hence it has a least mean error rate $(=0.142)$ for normal distributions. Maximum depth classifier based on likelihood depth has the least mean error rate $(=0.166)$ among the depth classifiers, which is competitive with QDA and SVM (with mean error rate =0.148). Maximum depth classifier based on Oja depth has the worst performance in this case. For bivariate Laplace distributions, Maximum depth classifier based on likelihood depth has the least mean error rate $(=0.255)$ among the depth classifiers, which is competitive with QDA (with mean error rate $=0.214$ ), SVM (with mean error rate $=0.214$ ) and $\mathrm{kNN}$ (with mean error rate $=0.282$ ). Mean error rates of other depth classifiers are a bit high. 


\section{DEPTH FUNCTIONS IN MAXIMAL DEPTH CLASSIFICATION RULES}

Presented in Table 3 is a comparison of computation time in seconds of each classifier when competing distributions are bivariate Laplace for one repetition. It is shown in Table 3 that QDA and $\mathrm{kNN}$ have the least computation time. However, computation time of maximum depth classifiers based on half-space depth, simplicial depth, Oja depth, zonoid depth and spatial depth are competitive with those of parametric classifiers.

\section{Analysis of Real Data}

A real dataset is also analysed to illustrate the performances of depth functions in maximal depth classification methods. Maximal depth classifiers are applied on mineral ions variability data. The data was extracted from a project experiment on crop science and production at the Institute for Agricultural Research and Training (IAR\&T) project titled "inter- and intra-maturity group differences in physiological quality of maize seeds" (Olasoji, 2014). The data contains measurements of mean amount of mineral ions ( $\mathrm{Na}, \mathrm{Ca}, \mathrm{K}$ and $\mathrm{P}$ ) leaked after 24 hours from soaked maize seeds at different maturity groups (early, intermediate and lately). Each observation consists of four attributes, which are mean mineral ions ( $\mathrm{Na}, \mathrm{Ca}, \mathrm{K}$ and $\mathrm{P}$ ). Each group consists of 36 observations. A random sample of size 30 and a test sample of size 6 are chosen. The experiment is repeated 100 times; quantile, mean and standard deviation of the proportions of misclassification associated with each of the classifiers are computed.

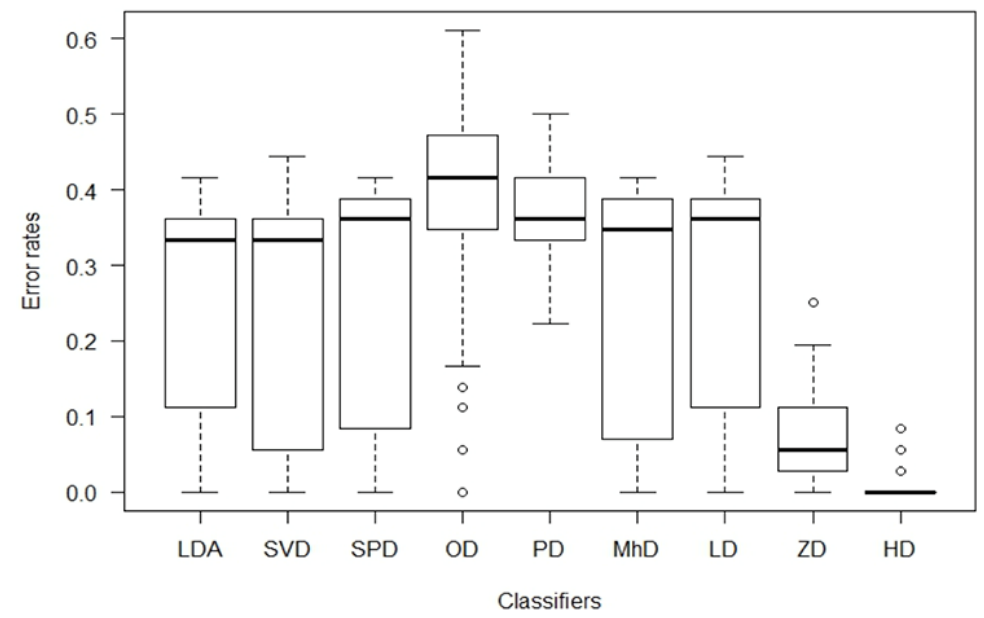

Figure 4. Box plot of proportions of misclassification associated with some classifiers for real data example 
Table 4. Quantiles, means and standard deviations of proportions of misclassification of some classifiers for real data example.

\begin{tabular}{lrrrrrrrrr} 
& \multicolumn{10}{c}{ Maximal Depth Classifiers } & \\
\cline { 2 - 9 } & HD & OD & SPD & SVD & PD & ZD & MhD & LD & LDA \\
\hline Minimum & 0.0000 & 0.0000 & 0.0000 & 0.0000 & 0.2222 & 0.0000 & 0.0000 & 0.0000 & 0.0000 \\
25\% Quantile & 0.0000 & 0.3541 & 0.0833 & 0.0556 & 0.3333 & 0.0278 & 0.0764 & 0.1111 & 0.1111 \\
Mean & 0.0069 & 0.3861 & 0.2697 & 0.2364 & 0.3717 & 0.0725 & 0.2683 & 0.2767 & 0.2619 \\
Median & 0.0000 & 0.4167 & 0.3611 & 0.3333 & 0.3611 & 0.0556 & 0.3472 & 0.3611 & 0.3333 \\
75\% Quantile & 0.0000 & 0.4722 & 0.3889 & 0.3611 & 0.4167 & 0.1111 & 0.3889 & 0.3889 & 0.3611 \\
Maximum & 0.0556 & 0.6111 & 0.4444 & 0.4444 & 0.5000 & 0.2500 & 0.4167 & 0.4167 & 0.4167 \\
Standard deviation & 0.0150 & 0.1478 & 0.1575 & 0.1618 & 0.0623 & 0.0600 & 0.1533 & 0.1462 & 0.1472 \\
\hline
\end{tabular}

Presented in Figure 4 is a comparison of maximum depth classifiers with linear discriminant analysis based on the proportions of misclassification using box plot. The figure shows that the maximum depth classifiers based on halfspace depth and zonoid depth has the least proportions of misclassification while the maximum depth classifiers based on Oja depth and projection depth has highest proportions of misclassification.

Presented in Table 4 is the quantile, mean and standard deviation of the proportions of misclassification associated with each of the competing classifiers. Maximum depth classifier based on half-space depth has the least mean proportion of misclassification as shown in the table. Use of spatial depth, simplicial volume depth, Mahalanobis depth and likelihood depth in maximum depth classifiers perform equivalently to LDA, while maximum depth classifiers based on half-space depth and zonoid depth outperform LDA. Simplicial depth values could not be computed as $d=4>2$. For computation of half-space depth, an approximate algorithm implemented in $R$ Package depth is used.

\section{Conclusion}

The maximum depth classifiers based on the training samples when any of the half-space depth, projection depth, simplicial depth, spatial depth, Oja depth, and majority depth is used, do not depend on any distributional assumptions or do not require any estimation of model parameters. That gives maximum depth classifiers an importance over parametric methods. Maximum depth classifiers are easily lent to multiclass cases. We have noted in our real data examples that the maximum depth classifiers are quite competitive with similar classifiers, especially when any of half-space or zonoid depth is used. 


\section{DEPTH FUNCTIONS IN MAXIMAL DEPTH CLASSIFICATION RULES}

\section{Acknowledgment}

The authors will like to thank Dr. J. Olasoji of the Institute for Agricultural Research and Training (IAR\&T), Ibadan for making mineral ions variability data available.

\section{References}

Anderson, T. W. (1984). An introduction to multivariate statistical analysis. NY: John Wiley \& Sons, Inc.

Chan, Y. \& Hall, P. (2009). Scale adjustments for classifiers in highdimensional, low sample size settings. Biometrika, 96(2), 469 - 478. doi: 10.1093/biomet/asp007

Cortes, C. \& Vapnik, V. N. (1995). Support-vector networks. Machine Learning, 20(3), 273 - 297. doi: 10.1007/bf00994018

Cover, T. M. \& Hart, P. (1967). Nearest neighbor pattern classification. IEEE Transactions on Information Theory, 13(1), 21 - 27. doi:

10.1109/tit.1967.1053964

Cuesta-Albertos, J. A. \& Nieto-Reyes, A. (2010). Functional classification and the random Tukey depth. Practical issues. Advances in Intelligent and Soft Computing, 77, 123 - 130. doi: 10.1007/978-3-642-14746-3_16

Donoho, D. L. \& Gasko, M. (1992). Breakdown properties of multivariate location parameters and dispersion matrices. The Annals of Statistics, 20(4), 1803 - 1827. doi: 10.1214/aos/1176348890

Dudoit S., Fridlyand, J. \& Speed, T. P. (2002). Comparison of discrimination methods for the classification of tumors using gene expression data. Journal of the American Statistical Association, 97(457), 77 - 87. doi: 10.1198/016214502753479248

Dutta, S. \& Ghosh, A. K. (2012). On classification based on $L_{p}$ depth with an adaptive choice of $p$. Technical Report No. R5/2011, Statistics and Mathematics Unit. Indian Statistical Institute, Kolkata, India

Dyckerhoff. R., Mosler, K. \& Koshevoy, G. (1996). Zonoid Data Depth: Theory and Computation. In: Prat, A. (Ed.) COMPSTAT: Proceedings in Computational Statistics 12th Symposium held in Barcelona, Spain, 1996. Berlin: Physica-Verlag HD. doi: 10.1007/978-3-642-46992-3_26 
Fisher, R. A. (1936). The use of multiple measurements in taxonomic problems. Annals of Eugenics, 7(2), 179 - 188. doi: 10.1111/j.1469-

1809.1936.tb02137.x

Fraiman, R., Meloche, J., García-Escudero, L. A., et al. (1999). Multivariate L-estimation. Test, 8(2), pp. 255 - 317. doi: 10.1007/bf02595872

Gao, Y. (2003). Data depth based on spatial rank. Statistics \& Probability Letters, 65(3), 217 - 225. doi: 10.1016/j.spl.2003.06.003

Ghosh, A. K. \& Chaudhuri, P. (2005). On maximum depth and related classifiers. Scandinavian Journal of Statistics, 32(2), 327 - 350. doi:

10.1111/j.1467-9469.2005.00423.x

Hall, P., Titterington, D.M. \& Xue, J. (2009). Median based classifiers for high dimensional data. Journal of the American Statistical Association. 104(488), 1597 - 1608. doi: 10.1198/jasa.2009.tm08107

Hubert, M. \& Van Driessen, K. (2004). Fast and robust discriminant analysis. Computational Statistics and Data Analysis, 45(2), 301 - 320. doi: 10.1016/s0167-9473(02)00299-2

Koltchinskii, V. I. (1997). M-estimation, convexity and quantiles. The Annals of Statistics, 25(2), 435 - 477. doi: 10.1214/aos/1031833659

Koshevoy, G. \& Mosler, K. (1997). Zonoid trimming for multivariate distributions. The Annals of Statistics, 25(5), 1998 - 2017. doi:

10.1214/aos/1069362382

Li, J., Cuesta-Alberstos, J. A. \& Liu, R. Y. (2012). DD-classifier: nonparametric classification procedure based on DD-plot. Journal of the American Statistical Association, 107(498), 737 - 753. doi:

10.1080/01621459.2012.688462

Liu, R. Y. (1990). On a notion of data depth based on random simplices. The Annals of Statistics, 18(1), 405 - 414. doi: 10.1214/aos/1176347507

Liu, R. Y. \& Singh, K. (1993). A quality index based on multivariate data depth and multivariate rank tests. Journal of the American Statistical Association, 88(421), 252 - 260. doi: 10.1080/01621459.1993.10594317

Liu, R. Y., Parelius, J. M. \& Singh, K. (1999). Multivariate analysis by data depth: Descriptive statistics, graphics and inference. The Annals of Statistics, 27(3), 783 - 858. doi: 10.1214/aos/1018031260

Mahalanobis, P. C. (1936). On the generalised distance in statistics. Proceedings of the National Institute of Sciences of India, 2(1), 49-55. 


\section{DEPTH FUNCTIONS IN MAXIMAL DEPTH CLASSIFICATION RULES}

Makinde, O. S. (2015). On some classification methods for high dimensional and functional data. $\mathrm{PhD}$ Thesis, University of Birmingham

Makinde, O. S. (2017). Multivariate rank outlyingness and correlation effects. Journal of Modern Applied Statistical Methods, 16(1), 246-260. doi: 10.22237/jmasm/1493597580

Makinde, O. S. \& Chakraborty, B. (2015). On some classifiers based on distribution functions of multivariate ranks. In Nordhausen, $\mathrm{K}$ and Taskinen, $\mathrm{S}$. (Eds). Modern Nonparametric, Robust and Multivariate Methods, Festschrift in Honour of Hannu Oja. NY: Springer, 249 - 264. doi: 10.1007/978-3-319-224046_15

Oja, E. (1983). Subspace methods of pattern recognition. Letchworth, England: Research Studies Press.

Olasoji, J. (2014). Inter- and intra-maturity group differences in physiological quality of maize seeds. Unpublished raw data.

Rousseeuw, P. J. (1984). Least median of squares regression. Journal of the American Statistical Association, 79(388), 871 - 880. doi:

10.1080/01621459.1984.10477105

Rousseeuw, P. J. \& Hubert, M. (1999). Regression depth (with discussion). Journal of the American Statistical Association, 94(446), 388 - 433. doi: 10.1080/01621459.1999.10474129

Rousseeuw, P. J. \& Van Driessen, K. (1999). A fast algorithm for the minimum covariance determinant estimator. Technometrics, 41(3), 212 - 223. doi: 10.1080/00401706.1999.10485670

Serfling, R. (2002). A depth function and a scale curve based on spatial quantiles. In Y. Dodge (Ed.). Statistical Data Analysis Based on the L1-Norm and Related Methods, pp. 25-38. Basel: Birkhau ser. doi: 10.1007/978-3-0348-82019_3

Tukey, J. W. (1975). Mathematics and the picturing of data. Proceeding of the International Congress of Mathematicians, Vancouver, 523-531.

Vapnik, V. N. (1998). Statistical learning theory. NY: John Wiley and Sons

Welch, B. L. (1938). The significance of the difference between two means when the population variances are unequal. Biometrika, 29(3-4), 350 - 362. doi: $10.2307 / 2332010$

Zuo, Y. \& Serfling, R. (2000a). General notion of statistical depth function. The Annals of Statistics, 28(2), 461 - 482. doi: 10.1214/aos/1016218226 


\section{MAKINDE \& ADEWUMI}

Zuo, Y. \& Serfling, R. (2000b). Structural properties and convergence results for contours of sample statistical depth function. The Annals of Statistics, 28(2), 483 - 499. doi: 10.1214/aos/1016218227 